R. A. Salerno and colleagues ${ }^{14}$ investigated the effect of D-pantothenyl alcohol on the function of the bladder as well as the gastro-intestinal tract. In 80 patients they found no difference between control and trial groups. They also gave the drug to 10 patients with established and complete ileus. This was a small series of ill patients in which controls, for obvious reasons, could not be used. They observed no effect within 24-36 hours, and all patients later responded with good effect to prostigmin. Although, as they pointed out, their trial was not statistically complete, they observed no beneficial effects from the drug and concluded that it was of no value either in the established severe cases of ileus or in influencing the post-operative course. They also suggested that, in fact, there was insufficient depletion of acetylcholine in these patients for the administration of even large doses of pantothenic acid to have any effect. Pantothenic acid itself has no cholinergic action.

Investigations so far have thus been inconclusive, but the impression is that while the administration of pantothenic acid may have some effect on post-operative gastro-intestinal motility this is not great enough to justify its routine use, and it is by no means the answer to complete and severe ileus. Post-operative gastrointestinal motility is very difficult to assess in a form which will allow statistical analysis, but it would seem that there is room for further research into the properties of pantothenic acid in a carefully controlled trial.

\section{SUBACUTE THYROIDITIS OF DE QUERVAIN}

Clinically de Quervain's subacute thyroiditis is characterized by a painful thyroid swelling of rapid onset accompanied by malaise and fever in severe cases, together with lassitude, slight nervousness, palpitations, and loss of weight. ${ }^{1}$ The pain in the thyroid frequently radiates to the ears or mandible, and there is pain on swallowing which frequently leads to a mistaken diagnosis of pharyngitis ${ }^{2}$ unless the thyroid is purposely examined. When mild the disease may subside in two weeks, but in more severe cases the swelling and pain in the thyroid gland may migrate from one lobe to the other and symptoms may continue for more than nine months. In most patients the illness lasts about three months. On palpation the thyroid enlargement is slight or moderate and is very firm and exquisitely tender. In migratory cases a firm tender nodule appears first on one side and a few weeks later on the other. The main feature of this condition is its well-defined course, ${ }^{3}$ which ends in complete disappearance of the thyroid swelling and remission of all symptoms, with no residual deficiency of thyroid function except in the very rare cases marked by repeated exacerbations over some months. 4

Subacute granulomatous thyroiditis of de Quervain is distinct from Hashimoto's disease and is in no way connected with the autoimmune group of diseases. Its aetiology is not yet fully established, but the most plausible hypothesis is that it is a viral infection. This was postulated many years ago because this type of thyroiditis follows upper respiratory infections in one-quarter to one-third of the cases, groups of cases may occur together in one locality, ${ }^{5}$ and there is a certain seasonal and geographical aggregation of cases. Furthermore, both its reversible course and the intensity of its general symptomatology resemble other known virus infections. In 1955-6 an epidemic of mumps occurred in Israel during which 15 cases of typical de Quervain's thyroiditis were observed. Mumps antibodies were detected in the sera of these patients, and in two cases mumps virus was isolated from thyroid gland tissue cultured in the brain of baby mice. A further case of mumps thyroiditis was reported in Great Britain, ${ }^{8}$ but many sporadic cases examined for mumps and other virus antibodies produced negative results, and it is not known whether other viruses can infect the thyroid. G. Meachim and M. H. Young ${ }^{9}$ recently made a careful survey of the histology in six cases and again pointed out the complete distinction from lymphadenoid goitre. Like many other observers they noted the rarity of the disease in material obtained at surgical operations. Clinically, subacute thyroiditis is also very uncommon in hospital practice, and in one London teaching hospital less than one case a year has been seen on the average over the past ten years. The ratio of women to men is four to six females to one male. It is likely that many mild cases are incorrectly diagnosed or treated at home, since it is rare to see patients with less than three to four weeks' history in out-patient departments.

Results of tests of thyroid function are characteristic in de Quervain's disease. Owing to disruption of the thyroid acini by the infecting organism iodinated proteins gain access to the circulation and may cause signs of hyperthyroidism, such as a raised protein-bound iodine value and basal metabolic rate, and endogenous thyrotrophic hormone may be suppressed. This feature together with the acinar destruction leads to a complete suppression of the uptake of radioiodine for four to twelve weeks. In mild cases tests of the overall 24-hour thyroid uptake may remain within normal limits but the scintigram shows areas of reduced uptake over the affected parts of the gland. ${ }^{10}$ The erythrocyte sedimentation rate is considerably raised and the leucocyte count remains normal. Tests for thyroid auto-antibody show traces of antibodies to colloid constituents in about $50 \%$ of cases, ${ }^{11}$ though characteristically antibodies to the thyroid cellular component are completely absent. All tests for antibody become negative again when the patient recovers, and this transient slight autoimmunization is probably the result merely of the

\footnotetext{
[ ${ }^{1}$ De Quervain, F., and Giordanengo, G., Mitt. Grenz. geb. Med. Chir., 1936, 44, 12 Vanderlinde. R. J., and Milne, J., J. Amer. med. Ass., 1960, 173, 1799.

' Crile, G., Ann. intern. Med., $1952,37,519$.

I: Ivy, H. K., J. clin. Endorr., 1961, 21, 1384.

- Czerniak, P., and Harell-Steinberg. A., J. clin. Endocr., 1957, 17, 1448.

? Eylan, E., Zmucky, R., and Sheba, Ch., Lancet, 1957, 1, 1062.

- Felix-Davies, D., ibid., 1958, 1,880.

Meachim, G.. and Young, M. H., J. clin. Path., 1963, 16, 189

Mahaux, J., Henry, J., Nagel, M., and Chamla-Soumenkoff, J., Ann. Endocr. (Paris), 1960, 21, 751.

Doniach, D., and Roitt, I. M., in Clinical Aspects of Immunology, 1963. Ed. Gell, P. G. H., and Coombs. R. R. A. Oxford, Blackwell.

13 Higgins, H. P., Bayley, T. A., and Diosy, A., J. clin. Endocr., 1963, 23, 235.

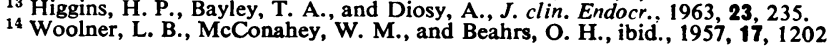


extensive leakage of thyroid antigens to which tolerance may be incomplete in the normal subject.

The differential diagnosis of de Quervain's thyroiditis from painful cases of autoimmune thyroiditis (lymphadenoid goitre) ${ }^{12}$ is based on several features. The onset of autoimmune thyroiditis is less acute and the uptake of radioiodine is normal or raised, while there are much higher titres of thyroid autoantibodies, particularly cellular microsomal antibodies detected by complement fixation.

The best treatment for patients with subacute thyroiditis is prednisone $15-20 \mathrm{mg}$. daily in cases with severe pain and pyrexia, and aspirin in mild cases. Recently the administration of triiodothyronine has been advocated ${ }^{13}$ in order to suppress secretion of thyroid-stimulating hormone (T.S.H.) more completely during the acute phase. Thyroidectomy is contraindicated as it may lead to myxoedema, ${ }^{14}$ and histological proof of the nature of the disease should be obtained by taking a thyroid-needle biopsy. Further progress in the understanding of this disease will be made by applying modern methods of virus culture to specimens of thyroid obtained at biopsy as soon as possible after the onset of the infection.

\section{TREATMENT OF HYDROCEPHALUS}

The obstructive type of hydrocephalus is due to obstruction of the pathways from the ventricles-by stenosis of the aqueduct and blockage of the foramina by which cerebrospinal fluid leaves the fourth ventricle. The non-obstructive (communicating) type is due to impaired absorption resulting from maldevelopment of the subarachnoid spaces or post-inflammatory obliteration of them. J. E. Scarff, of New York, a pioneer in the treatment of this intractable condition, has reviewed ${ }^{1}$ the whole story from W. E. Dandy's ${ }^{2}{ }^{3}$ early work on the secretion of cerebrospinal fluid in the ventricles, differentiating the two main types.

The classical operation for obstructive hydrocephalus was to form a new foramen as an outlet from the third ventricle. $^{4}$ Failure of absorption of cerebrospinal fluid was treated by destruction of the choroid plexuses. Dandy's ${ }^{6}$ original open technique had a mortality of $75 \%$, but T. J. Putnam ${ }^{7}$ and Scarff $^{8}$ devised endoscopic procedures with a lower mortality.

Interest was then aroused in procedures requiring the insertion of tubes to drain cerebrospinal fiuid from ventricles or spinal theca into a ureter, ${ }^{10}$ whence it

\footnotetext{
I Scarf, J. E., J. Neurol. Neurosurg. Psvchiat., 1963, $26,1$.

2 Dandy. W. E. and Blackfan, K. D., J. Amer. med. Ass., 1913, 61, 2216; Amer. J. Dis Child., 1914, 8406.

- Ann. Surg., 1919, 70, $129.1922,33,189$

Scarff. J. E., Arch. Neurol. Prvehiat. (Chic.), 1936, 35, 853.

- Darf. J. E., Arch. Neurol. Psvchiat. (Chic)

Putnam, T. J.. New Engl. J. Med., 1934, 210, 1373.

- Scarff. J E., J. Pediat. 1935, 6, 870.

- Matson. D. D.. J. Neurosurg., 1951, 8, 398.

10 Perlicurics, 1953,12,326.

11 Ransohoff, J., J. Neurosurg., 1954, 11, 295.

12 Scott M.. Wycis, H. T., Murtagh. F.. and Reyes, V., ibid., 1955, 12, 165.

18 Torkildsen. A., Arta chir. scand., 1939, 82, 117 .

14 Nulsen. F. E., and Spitz, E. B., Surg. Forum, 1952, 399

15 Callaghan. R. P., Cohen, S. J., and Stewart, G. T., Brit. med. J., 1961, 1, 860

16 Laurence. K. M., Lancet, 1958, 2, 1152.

- and Coates, S., Arch. Dis. Childh.. 1962, 37, 345.

Hadenius, A. M., Hagberg, B., HytNnas-Bensch, K., and Sjogren, I., Acta

puediat. (Upp vala), 1962, 51, 117

Childh., 1963, 38, 18 .
.
}

would be excreted, or into the serous cavities of pleura or peritoneum, whence it would be reabsorbed. ${ }^{11} 12$ Dehydration and loss of electrolyte were apt to complicate drainage into the ureter, while drainage into a serous cavity was sometimes followed by blockage of the tube owing to formation of a serous-lined envelope round it. A. Torkildsen's ${ }^{13}$ operation, which entails placing a tube from ventricle to cisterna magna, allows reabsorption by the natural route but is of limited application. More recently treatment with the Holter silicone valve has been developed. ${ }^{14}$ This device allows transference of cerebrospinal fluid from a lateral ventricle to the superior vena cava or atrium. It is a simple, safe technique applicable to all types of hydrocephalus at all ages, but has its own complications. The apparatus may become obstructed, and it has to be changed to allow for the child's growth. More important, chronic bacteriaemia from colonization of the valve occurs. ${ }^{15}$ Perhaps this risk would be met with in al] operations involving the use of foreign material.

Scarff surveys 618 cases treated by operations not requiring foreign materials and 1,087 treated by prosthetic methods. He finds that the mortality of $15 \%$ is the same in both groups, as is the initial success rate of $60-65 \%$, but that the incidence of severe late complications is 10 to 20 times greater after the shunting procedures with tubes or valves than after operations performed without these devices. But it seems doubtful whether the two groups of cases are properly comparable. Moreover the considerable spontaneous-arrest rate-about $40 \%^{16-18}$-is not taken into account. Indeed if the earlier operations were as satisfactory as appears it is difficult to see why such enthusiasm has been shown for the valves. Scarff makes an important point in stressing that the thickness of the pallium is no guide to the baby's intellectual potential and that some guide is badly needed.

The management of hydrocephalus is closely linked with that of spina bifida, and here too the old hopeless attitude is being challenged, for thousands of these babies are surviving for some kind of life which might have been a fuller one had treatment been given. W. J. W. Sharrard and colleagues ${ }^{19}$ have shown in a controlled trial that a great reduction in the degree of paraplegia can be obtained by closing the defect soon after birth. This prevents damage to the exposed neural tissue by drying, infection. and stretching of the nerves across the expanding sac. Furthermore it allows the baby to go home without the unpleasant swelling needing constant nursing attention, so that the mother can care for her baby at least for a while.

\section{THE ESMARCH BANDAGE}

The invention of the Esmarch bandage, first described in $1873,{ }^{1}$ post-dates the invention of the plaster-of-Paris bandage by a mere twenty years. Both serve the convenience of the surgeon, particularly the orthopaedic surgeon, so efficiently that it is impossible to imagine surgical practice to-day without them. Yet in both lie potential dangers. The risk of swelling of the tissues below a plaster and interruption of the circulation is well enough recognized, and an incomplete, padded, or split 